\title{
INTEGRAL-BASED FILTERING OF CONTINUOUS GLUCOSE SENSOR MEASUREMENTS FOR GLYCAEMIC CONTROL IN CRITICAL CARE
}

J Geoffrey Chase ${ }^{1}$, Christopher E. Hann ${ }^{1}$, Monique Jackson ${ }^{1}$, Jessica Lin ${ }^{1}$, Thomas Lotz $^{1}$, Xing-Wei Wong ${ }^{1}$ and Geoffrey M Shaw ${ }^{2}$

${ }^{1}$ Department of Mechanical Engineering, Centre for Bio-Engineering, University of Canterbury, Private Bag 4800, Christchurch, New Zealand, Email: geoff.chase@canterbury.ac.nz ${ }^{2}$ Department of Intensive Care, Christchurch Hospital, Christchurch School of Medicine and Health Science, University of Otago, Christchurch, New Zealand

\section{ABSTRACT}

Hyperglycaemia is prevalent in critical illness and increases the risk of further complications and mortality, while tight control can reduce mortality up to $43 \%$. Adaptive control methods are capable of highly accurate, targeted blood glucose regulation using limited numbers of manual measurements due to patient discomfort and labour intensity. Therefore, the option to obtain greater data density using emerging continuous glucose sensing devices is attractive. However, the few such systems currently available can have errors in excess of $20-30 \%$. In contrast, typical bedside testing kits have errors of approximately 7-10\%. Despite greater measurement frequency larger errors significantly impact the resulting glucose and patient specific parameter estimates, and thus the control actions determined creating an important safety and performance issue. This paper models the impact of the Continuous Glucose Monitoring System (CGMS, Medtronic, Northridge, CA) on model-based parameter identification and glucose prediction. An integral-based fitting and filtering method is developed to reduce the effect of these errors. A noise model is developed based on CGMS data reported in the literature, and is slightly conservative with a mean Clarke Error Grid (CEG) correlation of $R=0.81$ (range: $0.68-0.88$ ) as compared 
to a reported value of $\mathrm{R}=0.82$ in a critical care study. Using 17 virtual patient profiles developed from retrospective clinical data, this noise model was used to test the methods developed. Monte-Carlo simulation for each patient resulted in an average absolute one-hour glucose prediction error of 6.20\% (range: 4.97-8.06\%) with an average standard deviation per patient of 5.22\% (range: 3.26-8.55\%). Note that all the methods and results are generalisable to similar applications outside of critical care, such as less acute wards and eventually ambulatory individuals. Clinically, the results show one possible computational method for managing the larger errors encountered in emerging continuous blood glucose sensors, thus enabling their more effective use in clinical glucose regulation studies.

Keywords-Critical Care, Glucose, Hyperglycemia, CGMS, Parameter Identification

\section{INTRODUCTION}

Hyperglycaemia and high levels of insulin resistance are prevalent in critical care [14]. Nutritional support regimes with a high carbohydrate content often compound the counter-regulatory response and do not suppress endogenous glucose production as normal $[3,4]$. Inhibiting the response to increased glycemic levels are factors such as increased insulin resistance, absolute or relative insulin deficiency, and drug therapy. Although hyperglycemia can be a marker of severity of illness, it can also worsen outcomes, leading to an increased risk of further complications such as severe infection [5], myocardial infarctions [1], polyneuropathy and multiple-organ failure 
[2]. Tight glucose control has been shown to reduce ICU patient mortality by as much as $43 \%[2,6]$.

To better control glucose levels, model-based adaptive control methods [e.g. 7,13,1819] and sliding-scale protocols [e.g. 8] have been tested. Model-based methods can be very accurate, but require the ability to identify patient specific parameters and capture all of the observed dynamics. Chase et al [7] used a convex, computationally efficient integral-based formulation presented by Hann et al [9], which had the benefit of filtering the glucose errors encountered. However, that work used clinical data from GlucoCard bedside testing kits with an average error of $7-10 \%$, whereas currently available emerging continuous glucose systems can have much larger errors for any given measurement.

Chase et al [7] measured blood glucose every 30 minutes to achieve tight control. Due to the discomfort and labour intensity of such frequent measurement, emerging semiinvasive continuous glucose sensors offer several advantages. In particular, they offer much higher data density, ranging from every 5 minutes to every 20 minutes [10-12], without any significant clinical effort. This higher data density could significantly improve the ability of model-based control methods to better fit patient specific parameters and more quickly react to sudden changes in patient condition.

For example, Figure 1 shows a 24-hour critical care clinical trial using the adaptive control methods from Chase et al [7], but measuring only every hour. At $~ 180-200$ minutes the patient experienced a significant atrial fibrillation episode. Such episodes are preceded by a surge of counter-regulatory hormones, such as adrenaline, which is 
identified by the controller at $\sim 120$ minutes as a major, sudden drop in modelled insulin sensitivity, $S_{I}$. However, despite the relatively early warning, the controller could not react fast enough to fully prevent glucose levels from rising due, in part, to the relatively infrequent hourly measurement required for patient comfort and to minimise clinical labour.

A much greater number of measurements, as might be obtained from emerging continuous glucose sensors, would provide the data to more readily identify, and react to, sudden changes in patient condition. Thus, more frequent measurement could be used to capture rapid changes in patient specific metabolic parameters. Identifying such parameters would enable accurate prediction of the impact of insulin or nutrition interventions, which is the critical element in any glycaemic control approach [7]. Hence, it is thus the sudden change in $S_{I}$ that this research aims to identify more rapidly and accurately using frequent measurement from emerging glucose sensors. The main requirement is enough accuracy to ensure accurate identification of the underlying modelled metabolic parameters that allow accurate model prediction of the affect of different clinical interventions. Therefore, this example is presented to illustrate the potential clinical control and patient benefits that could be obtained with frequent, accurate measurement.

\section{[Insert Figure 1 here]}

The Continuous Glucose Monitoring System (CGMS, Minimed, Northridge, CA) currently offers the greatest data density with little difference in error from other reported emerging sensor technologies, and has been approved for clinical use by the 
FDA in the US [11,12]. While this technology is new, it has been evaluated in critical care [11], and been used for automated glucose regulation in critical care [13]. However, due in part to the errors and accuracy encountered, both reports were less than fully successful. CGMS sensors were also recently evaluated for glucose regulation in surgical units where the conclusion was that some technical and accuracy improvements were required before they could be regularly used to monitor strict glycaemic control [14].

It is important to note that CGMS sensors have been initially developed as complementary measurement systems, rather than replacements for current glucose pin-stick methods [15], and are very effective at capturing trends and the impact of therapy changes. Current measurements of sensor error, such as the Clarke Error Grid (CEG) further illustrate this point [16]. The CEG is used to assess sensor accuracy by plotting the sensor measurement versus a gold-standard, usually laboratory, glucose measurement. The CEG also is broken into grids labelled A-D to assess the impact of the resulting sensor accuracy on the patient's clinical treatment response [16], where the A and B zones are considered clinically accurate enough not to have significant negative impact on the likely clinical treatment response. However, the CEG assessment allows for larger errors of 20-30\%, than might be desired for computational model parameter fitting and semi-automated, or automated, feedback control systems.

The main advantage of automated, regular glucose measurements is their potential in tightly regulating blood glucose levels in treatment, both in hospital and for ambulatory individuals. Such automated solutions have been the topic of significant 
recent discussion [e.g. 17-19], in part due to the emergence of sensors like the CGMS. These discussions primarily noted two things. First, that these automated sensors could be readily linked to a control loop as they remove manual clinical effort from the existing bedside glucose measurement process. Secondly, they noted that the measurements obtained were accurate enough to be potentially useful in clinical control situations [18-19]. Additionally, there has already been a study in a critical care unit [13], which represents a high acuity ward where automation might be most likely to first appear. The primary requirement is measurements with error low enough to minimise estimation errors that could bias clinical treatment decisions from an automatic controller.

It is also important that any filtering or estimation based on a noisy measurement does not have significant time lag due to the method used. Minimising lag in the filtered/estimated glucose levels used in model-based control is critical in ensuring that subsequent glucose predictions are accurate. Without minimising lag, the modelled state for determining control action might not fully represent the actual state, leading to potentially unsafe clinical control actions and poor outcome.

Therefore, this paper develops integral-based fitting and filtering methods to reduce the impact of larger errors and noise from these sensors. The goal is to produce a minimum lag estimate with good accuracy. The performance measure is expressed as mean absolute prediction error 1-hour ahead, and is used to assess the methods presented. This model prediction performance measure represents the fitted models ability to use the measured glucose data to accurately identify patient specific model parameters so that it can then be used to determine the affect of interventions, and 
thus provide good control. Note that many similar semi-invasive sensors are emerging and have similarly wide ranges of potential error. To be clinically effective, particularly for glycaemic control applications, the larger errors that occur must be readily managed by consistent, programmable methods to take full advantage of the higher data density and lower clinical labour requirements returned by these sensors.

\section{METHODOLOGY}

\section{A. Physiology and System Model}

The system model employed has been presented and validated in Chase et al [7] and related clinical studies [20-22]. This model provides the basis for the filtering and fitting methods developed.

$$
\begin{gathered}
\dot{G}(t)=-p_{G} G(t)-S_{I}\left(G(t)+G_{E}\right) \frac{Q(t)}{1+\alpha_{G} Q(t)}+P(t) \\
Q(t)=k \int_{0}^{t} I(\tau) e^{-k(t-\tau)} d \tau \\
\dot{I}(t)=-\frac{n I(t)}{1+\alpha_{I} I(t)}+\frac{u(t)}{V_{I}}
\end{gathered}
$$

where $G(t)=$ concentration of the plasma glucose above the equilibrium level $(\mathrm{mmol} / \mathrm{L}), G_{E}=$ equilibrium level of plasma glucose concentration $(\mathrm{mmol} / \mathrm{L}), I(t)=$ concentration of the plasma insulin (mU/L), $P(t)=$ exogenous glucose appearence rate $(\mathrm{mmol} /(\mathrm{L} \cdot \mathrm{min})), u(t)=$ insulin infusion rate $(\mathrm{mU} / \mathrm{min}), Q(t)=$ interstitial insulin concentration $(\mathrm{mU} / \mathrm{L}), I(t)=$ plasma insulin concentration $(\mathrm{mU} / \mathrm{L}), V_{I}=$ assumed insulin distribution volume (L), $n=$ delay in interstitial transfer of insulin $\left(\min ^{-1}\right), p_{G}=$ time-varying fractional clearance of plasma glucose at basal insulin $\left(\mathrm{min}^{-1}\right), S_{I}=$ time- 
varying insulin sensitivity $(\mathrm{L} / \mathrm{mU} \cdot \mathrm{min}), k=$ parameter controlling the effective half life of insulin $\left(\mathrm{min}^{-1}\right), \alpha_{I}=$ Michaelis-Menten parameter for insulin transport saturation, $\alpha_{G}=$ Michaelis-Menten parameter for glucose clearance saturation.

The model accounts for non-linear saturation of exogenous insulin disappearance rate from plasma (in Equation (3)) and its saturable utilisation to reduce blood glucose levels (in Equation (1)). The addition of transient insulin kinetics through interstitial boundaries via a convolution integral accounts for the dynamics seen in clinical trials [7], and better matches physiological knowledge. This model therefore effectively splits the glucose compartment into fast and slow compartments over a continuum rather than discrete states (e.g. [23]). Finally, significant exogenous insulin infusions, as typically encountered in hyperglycaemic critical care patients, effectively suppress endogenous insulin production [24], which is also modelled here as captured by the term $p_{G}$ for simplicity $[7,9]$.

\section{B. Modeling Noise for the CGMS Sensor}

All the available literature reports CGMS accuracy and error referenced to laboratory standard measurements using a Clarke Error Grid. Hence, the exact distribution of the error is unknown. To add this error, an approximate model was created using the data available. Specifically, $78 \%$ of the measurements are within $20 \%$ of the actual value, defining the A range on the Clarke Error Grid [25], and the correlation between actual and the approximated blood glucose values is around 0.88 [11]. In addition, maximum errors in the literature are no greater than $40 \%$ based on observation of the results presented. These reported error values are also noted throughout the literature on 
these sensors [12-20] and create an overall picture suitable for a simple, approximate model.

Hence, the error can be simply and approximately modelled using a normal distribution with $17 \%$ (0.17) standard deviation. This standard deviation and distribution allows $78 \%$ of the measurements to be within $20 \%$, matching the reported values of [11]. In addition, a limit of 40\% ( 2.5 standard deviations) was put in place to limit the peak error to match the reported data as observed in [10-20]. Thus, the overall model is quite simply a normally distributed random noise added to a simulated glucose value.

Note that none of the references [10-20] provides specific statistical distributions or histograms to provide further insight, as all errors are primarily reported for this and similar sensors in terms of Clarke Error Grid performance. In addition, the Clarke Error Grid, while it does not show the resulting error distribution, is the current gold standard for reporting such sensor performance. Figure 2 shows a segment of a simulated patient glucose profile with " $\mathrm{x}$ " denoting the value with added CGMS error. Bands are shown for $20 \%$ and $40 \%$ error to show the size of the error that can occur using the resulting model.

\section{[Insert Figure 2 here]}

\section{Parameter Fitting and Identification}

The parameters $\left(V_{I}=12 \mathrm{~L}, n=0.16 \mathrm{~min}^{-1}, k=0.0099 \mathrm{~min}^{-1}, \alpha_{G}=0.04 \mathrm{~L} / \mathrm{mU}, \alpha_{I}\right.$ $=0.0017 \mathrm{~L} / \mathrm{mU}$ ) are held constant at mean values found through an extensive literature 
search $[9,26]$. The exogenous feed details, $P(t)$, are known for each patient in this study, and would also be known for critical care enteral feeding regimes.

The parameter identification method is integral-based similar to Hann et al [9] and identifies $S_{I}$ as a time varying value, holding $p_{G}$ constant per the sensitivity study results from that research. In this research, the equilibrium glucose level, $G_{E}$, is also identified as it can vary significantly as condition evolves. This approach identifies the major dynamics of hyperglycaemic patients, as primarily driven by insulin sensitivity, before secondary parameters are modified if required. Insulin sensitivity is the critical parameter as its effective value in this critical care model can evolve significantly over time with patient condition and drug therapy [3-4,7,9,21,26].

It is therefore important to ensure the fitting method for identifying time varying patient specific parameters is as low in computational effort as possible, so other parameters can be varied without significantly affecting the overall computation time. Computational time is a significant factor to consider in real time control, as well as in the process of refining and testing the model on large numbers of patients.

The integral based method presented is developed as follows. First, integrating both sides of Equation (1) and defining $\bar{Q}=Q /\left(1+\alpha_{G} Q\right)$, the following expression holds for any segment of time from $t_{0}$ to $t$ :

$$
\begin{gathered}
\int_{t_{0}}^{t} \dot{G} d t=\int_{t_{0}}^{t}\left(-p_{G} G-S_{I}\left(G+G_{E}\right) \bar{Q}+P\right) d t \\
\Rightarrow G(t)-G\left(t_{0}\right)=-\int_{t_{0}}^{t} p_{G} G d t-\int_{t_{0}}^{t} S_{I}\left(G+G_{E}\right) \bar{Q} d t+\int_{t_{0}}^{t} P d t
\end{gathered}
$$


Substituting the total glucose level $G_{T}=\left(G+G_{E}\right)$ into Equation (4) results in an equivalent expression that is easy to compute given measured total glucose levels:

$$
G_{T}(t)-G_{T}\left(t_{0}\right)=-\int_{t_{0}}^{t} p_{G}\left(G_{T}-G_{E}\right) d t-\int_{t_{0}}^{t} S_{I} G_{T} \bar{Q} d t+\int_{t_{0}}^{t} P d t
$$

To reduce computational complexity and account for a variation over time, the total time interval considered is divided into segments over which $S_{I}$ is piecewise constant.

$$
S_{I}=\sum_{i=1}^{N} S_{I, i}\left(H\left(t-t_{(i-1)}\right)-H\left(t-t_{i}\right)\right)
$$

where $H\left(t-t_{0}\right)$ is the Heaviside function defined $H\left(t-t_{0}\right)=0$ when $t$ is less than $t_{0}$, and $H\left(t-t_{0}\right)=1$, when $t$ is greater than or equal to $t_{0}$. Note that $N$ in Equation (6) may be different depending on the number of hours used per segment. For this research, the glucose effectiveness, $p_{G}=0.01$, is held constant based on prior sensitivity analysis results [9]. The advantage of fixing $p_{G}$ is that the parameter $G_{E}$ can be included as an extra variable to be identified. This parameter is not easily measured in critical care and was approximated by moving averages in Hann et al [9].

Using Equation (6), Equation (5) can be rewritten and expanded:

$$
G_{T}(t)-G_{T}\left(t_{0}\right)=-\int_{t_{0}}^{t} p_{G} G_{T} d t+p_{G} G_{E}\left(t-t_{0}\right)-\left(\sum_{i=1}^{i=j-1} S_{I, i} \int_{t_{0}}^{t_{0}+60 i} G_{T} \bar{Q} d t+S_{I, j} \int_{t_{0}}^{t} G_{T} \bar{Q} d t\right)+\int_{t_{0}}^{t} P d t
$$


where the parameter $G_{E}$ is now a linear unknown variable along with the $S_{I, i}$ terms defining insulin sensitivity, and the integrals are evaluated over $t_{0}+60(j-1)<t<$ $t_{0}+60 j$ and $j=1, \ldots, N$. However, these constants can be factored out after numerically integrating the data, resulting in a convex linear system of equations.

$$
\underline{A}\left\{\begin{array}{l}
S_{I i} \\
G_{E}
\end{array}\right\}=\underline{b}
$$

where the number of equations for each time segment can be arbitrarily selected by integrating over different time periods within that segment. To ensure solution values within physiologically valid ranges, weighted constraints are employed [7,9].

The convex least squares solution of Equation (8) defines the time-varying profile of $S_{I}$ for that time period. Using integrals, instead of derivative based fitting methods, has the advantage of being robust to noise in the measured glucose data, effectively providing a low-pass filter in the summations involved in numerically integrating the data. A full error analysis is contained in Hann et al [9] along with further details similar to both methods.

\section{Modified Method for CGMS}

In Chase et al [7] three GlucoCard glucose measurements are used with linear or higher order approximations to fit $S_{I}$ over sequential 1-hour periods during clinical glucose control trials. The GlucoCard is one example of several similar bedside 
glucose test kits that use a pin-stick and capillary blood, or (if available [7]) arterial blood via a catheter, to obtain a sample that is manually inserted into a calibrated device via a test strip. It should be noted that in transient cases, such as after a meal, there can be up to 5-20 minutes of lag between whole blood measurements and the subcutaneous measurements from CGMS and similar sensors. In the context of this research, GlucoCard measurements assumed only 7\% error and therefore did not overly bias approximated glucose curve used to fit $S_{I}$. This limited effect is also partly due to the lower number of measurements, as only three (3) are available over the 1hour period, that could potentially significantly bias the results. Overall, the use of GlucoCard measurements at 30-60 minute intervals versus subcutaneous measurements with 5-20 minute lag at 5 minute intervals represents, qualitatively, an even to improved tradeoff for using the more frequent measurement.

Figure 2 shows a glucose curve with CGMS noise approximated as defined. This curve shows the wide spread and larger errors that can be encountered with this type of sensor. If 13 measurements are available per hour (every 5 minutes) and 2-4 are significantly biased in one direction, then integrating over periods of up to 2 hours will not be long enough to average out their effect given the size of the potential errors of up to 20-30\%. Hence, directly applying the methods of [9] for clinical adaptive glucose control, as in [7], may lead to inaccurate parameter identification.

A solution to this problem is to use longer periods of data to perform the fitting, as well as smoothing the results of the parameter identification. The effect of a group of significantly biased measurements will then be effectively averaged over a longer period using integral methods, but without the lag introduced by long, higher order 
filters. Thus, the methods of [7] can be reformulated for integrations across longer periods, with fixed base point $t_{0}$ in Equation (4) for that period. This approach preserves the convex solution, global minimum and lower computational intensity of the overall approach.

More specifically, a rolling 12-hour period is employed and the identified insulin sensitivity profile, $S_{I}(t)$, is smoothed to add additional filtering. The smoothing is defined as a modified moving average of the 12 hourly insulin sensitivity values determined every hour.

$$
\begin{aligned}
& S_{I, 1}=\left(4 S_{I, 1}+2 S_{I, 2}+S_{I, 3}\right) / 7 \\
& S_{I, k}=\left(S_{I, j-1}+S_{I, j}+S_{I, j+1}\right) / 3 \\
& S_{I, 12}=\left(S_{I, 10}+2 S_{I, 11}+4 S_{I, 12}\right) / 7
\end{aligned}
$$

where $S_{I, j}$ is the identified insulin sensitivity parameter value for hour $j=1 \ldots 12$ obtained from Equation (8), and $j=12$ is the current time value of insulin sensitivity that is used to predict blood glucose variation over the next hour. The middle term of Equation (9) is a standard 3-point moving average and the end terms are designed to weight the end value the most, while reducing bias that can occur if an outlying measurement error occurs.

This smoothing step is similar in concept to applying a second integration of Equation (5), but with specific weightings for the end values in the 12-hour window. To start the algorithm the initial periods during the first 12-hours are done the same way, but over the shorter time frame. As a result, there is a new prediction of $S_{I}$ each hour to 
predict blood glucose levels in the subsequent hour. The overall process is shown in Figure 3 and has the following specific steps:

- Obtain data over 12-hour window (less if starting)

- Numerically integrate as in Equation (5)

- $\quad$ Solve for $S_{I}(t)$ using Equation (8)

- Smooth results with Equation (9)

\section{[Insert Figure 3 here]}

Note that while this method shares the same formulation as [9] it has three major differences. First, it extends the method to integrate over longer periods, in this case 12 hours, with a fixed base point, and uses the added smoothing defined in Equation (9) to avoid the impact of outlying measurement errors to overly bias the fitted insulin sensitivity and subsequent glucose prediction. Second, it is designed to run forward in time, rather than retrospectively over an entire data set, as would be encountered in clinical glycaemic control applications. Finally, the use of an integral formulation over such a long time interval, versus very high order filters, ensures that no significant lag is added, except via the three point smoothing of Equation (9), without needing to use a model for the noise or the sensor dynamics.

\section{E. Patient Data and Selection Criteria}

To test this method, a random selection of 17 patients from a 201 patient data audit at Christchurch Hospital were selected [26]. Each patient record had a period greater than one day with intervals between measured data points of 3 hours or less. The data 
density of three hours was selected to ensure enough measurements to enable a good model evaluation. The entire length of stay was not always considered, as many patients only had a shorter period of data that fitted the criteria. This subset broadly represents the cross section of patients seen in the ICU, regarding medical condition, age, sex, APACHE II scores and mortality, as summarised in Table I. Type 1 and Type 2 diabetes is somewhat over-represented because these patients are often more frequently measured. Note that BMI is not typically recorded in most ICU's and was therefore not retrospectively available. Patients with serious head injury, morbid obesity, or who were moribund, were excluded. Ethical consent was obtained from the Canterbury Ethics Committee for this retrospective data analysis and research.

\section{F. Method Validation and Testing}

Each patient data set had already been fitted [9] and a time-varying $S_{I}(t)$ profile and approximated trajectory for GE were available. This data creates a virtual patient from which a glucose profile can be obtained using the retrospective feed and insulin infusion details, or other inputs as desired. Normally distributed CGMS sensor noise is added, as described, to the resulting virtual patient glucose profiles and the methods developed are then applied to identify $S_{I}$ and $G_{E}$. These parameter values are then used in the model to predict subsequent blood glucose levels one hour later. The entire process mimics the control algorithm used in clinical adaptive control of glucose, such as presented in [7].

Each patient profile was simulated $\mathrm{N}=20$ times to test the algorithm and generate statistics on performance. Such, repeated Monte Carlo style simulation allows appropriate performance statistics to be generated. Thus, there are $17 * 20=340$ 
simulations in total where each is different due to the use of random added noise given to each simulated glucose measurement. The outcome performance measurement is a comparison of the predicted blood glucose over the next hour and the simulated noise-free "true" value. Low mean absolute prediction error indicates successful filtering of the measurement error and accurate identification of the modelbased insulin sensitivity at that point in time. The overall goal is an analytical proof of concept test of the methods developed prior to clinical testing.

\section{RESULTS}

Figure 4 shows the Clarke Error Grid for a simulation of one of the 17 patients, and the correlation coefficient of $\mathrm{R}=0.82$ for all $\mathrm{N}=20$ simulations. The results are visually comparable to those found in the literature and the correlation coefficient is slightly lower than reported values for critical care evaluation of 0.88 [11]. The only major difference to figures seen in the literature is the clear $40 \%$ maximum error limitation that is visible for the extreme points in Figure 4. Finally, note that each individual patient simulation yielded 500-2000 simulated measurements. Hence, the error grids, as shown in Figure 4, are primarily useful qualitatively due to the extreme number of points shown.

Table II shows the correlation coefficients obtained from 7 patient profiles over $\mathrm{N}=$ 20 simulations. The average correlation coefficient obtained for all 20 simulations over all 7 patients was 0.81 [range: $0.68-0.88$ ]. Only 7 randomly selected patients, out of 17 , were used to evaluate the noise model to validate the fundamental assumptions. The reason for using only 7 is that over 20 simulations of average 3 days length of 
stay, each patient thus generates approximately 17,000 random simulated measurements, which is more than adequate for a simple validation of the assumed error model presented. Analysis of the remaining 10 patients did not change range or average values reported.

\section{[Insert Figure 4 here]}

The results in Figure 4 and Table II show that the simple, approximate error model employed is slightly conservative. More specifically, the correlation coefficients are approximately $8 \%$ lower and only the maximum was equal to that reported in the literature. These differences are likely due to the actual CGMS error distribution being slightly tighter than a true normal distribution as shown schematically in Figure 5. Thus, more of the errors in the $20 \%$ band would be distributed closer to the mean value than the normal distribution allows. This result is further confirmed by the mean absolute relative difference (MARD) reported in CGMS trials of 13\% [27] being slightly lower than the $17 \%$ standard deviation value used in the model.

\section{[Insert Figure 5 here]}

Overall, the simple, normally distributed error model proposed is a fairly close approximation to the limited clinical data reported. It also represents a conservative estimate of the error with respect to testing fitting and filtering methods by slightly overstating the magnitude of the errors that might be observed. Therefore, it is used for further analysis as is in this research. 
Table III presents the mean one hour (forward) prediction errors for all 17 patients, where forward predictions were made every hour of the record after fitting the model parameters. The inputs used were the retrospective hospital record insulin and nutritional infusions. The standard deviation of those errors is reported to indicate the variance. Table III also includes the same results for the 10 patients similarly considered in Hann et al [9], where 30-minute GlucoCard measurements with 7\% error were assumed. Comparing the results yields the impact of the greater number of measurements obtained with CGMS, the impact of its larger magnitude errors, and the impact of the methods presented to smooth the resulting glucose data so that accurate model fits and glucose response predictions are obtained.

\section{[Insert Table III here]}

Table III shows that the average error with the methods presented is $6.20 \%$, which is effectively the same as the GlucoCard results. The standard deviations of that error of $5.22 \%$ and $5.31 \%$ respectively, are also effectively identical. Hence, the methods presented are effective at providing predictive performance equal to that obtained with the lower error GlucoCard bedside testing kits which have been shown to yield good results in simulation [9] and in clinical control [7].

More specifically, these results show that the filtering and resulting identification of patient specific metabolic parameters $\left(S_{I}\right)$ resulted in successful prediction by reducing the effect of the larger CGMS errors on fitting and prediction. These results are thus a comparison between the Monte Carlo simulated and filtered data, followed by identification and prediction, and the clinically recorded data and fitted value from 
[9]. In particular, it should be noted that the modelled noise distribution used to approximate CGMS error is much wider than the resulting prediction noise, although both are normally distributed, indicating the overall efficacy of the methods presented through the steps of identification and control prediction. However, it should be noted that only dynamics within the model are captured in this proof of concept study and clinical testing where unmodelled dynamics may occur will be necessary.

The worst case error however is much lower at $8.06 \%$ versus $10.90 \%$, indicating more consistent performance and possibly the positive effect of greater numbers of measurements. For both sensors this worst case result occurs for Patient 554, indicating that this patient is equally difficult for both methods and that part of the worse results seen may be due to the model used for both cases not fully capturing this particular patient's dynamics. For the best case, Patient 1090’s GlucoCard results are significantly better, showing that in this case, the lesser number, lower error measurement system still had an advantage. Finally, it is worth repeating that the average errors across the cohort were very similar, indicating that with the methods presented, both systems would likely have similar performance overall.

An overall premise of this research is that directly using the methods of Hann et al [9] with a short window on CGMS data would result in potentially large prediction errors. Examining Patient 24 and applying the methods of [9] directly results in an average absolute prediction error of $18.25 \%$ with a standard deviation of $13.11 \%$, indicating that a significant number of errors will be greater than $20-30 \%$. These errors are large enough that clinically unsafe decisions might be taken by a controller. 
In comparison, the methods presented resulted in an average absolute prediction error of $4.97 \%$ with standard deviation of $3.89 \%$, which is $3-4$ times lower. Note that approximating each hours worth of measurements with a linear line to reduce the effect of outliers, and then applying the methods of [9] directly, still results in an average absolute prediction error of $10.37 \%$ with standard deviation of $7.11 \%$. This last result is still effectively 2 times larger than both the GlucoCard and current results presented for that patient. This example reinforces the overall concept that longer integration intervals, along with the smoothing method presented, are required to provide enough filtering to obtain prediction results with minimal lag that are suitable for clinical adaptive control applications.

\section{DISCUSSION}

The method presented uses the virtual patient data and extends the integral-based methods from Hann et al [9] to reduce the impact of noise in glucose prediction for clinical control applications similar to those in Chase et al [7]. Therefore, it represents only one way to manage this data and measurement error. Other approaches may provide equally good results.

There is a large body of work on digital filtering that could be applied to this problem. While many digital filters can provide a clear, accurate signal from similarly noisy data, they can also introduce significant lag. More specifically, $4^{\text {th }}$ to $8^{\text {th }}$ order IIR filters have been successfully applied to this data. However, to obtain a similar $\sim 7 \%$ accuracy in glucose value results in 30-40 minutes lag between predicted values and (simulated) true values. For clinical control applications in critical care, where patient 
condition, insulin resistance and glucose levels can evolve rapidly over this time frame, this lag could prove unacceptably large for tight control.

More complex filters based on Kalman or H-infinity theory may also be applicable. These approaches require, in the linear cases, a linear model of the system, which will not capture the range of dynamic behaviours seen in critical care that are necessary for providing tight control $[7,13,20]$. For non-linear methods a model is still required for which the validated system model presented may not be tractable for filter design. Recently, Knobbe and Buckingham [28] used non-linear Extended Kalman Filtering with CGMS and reference blood glucose data, and obtained a mean average deviation (MAD) of 9.6-16\%, which is larger than the results presented. It should also be noted that this data was for ambulatory individuals and not critical care patients. However, such an approach could offer similar performance with a trade off of greater computational and design effort as model and filter complexity increases.

Finally, the current CGMS monitor offers analysis software that can filter the data. However, this software uses windows up to 72 hours long to retrospectively fit the glucose data. In this research, the goal is to fit patient specific parameters, a process that is sensitive to large error or individual outliers, and then predict glucose accurately. Hence, the fitting methods used in that software are not necessarily directly applicable for a real-time application.

The method presented proves very effective in reducing a typically non-linear, nonconvex optimization problem to a simple convex, linear system. All fitted values are within physiologically valid ranges reported in the literature. The integral method is 
shown to be very fast and offers significant computational speed advantages over nonlinear approaches [9]. As presented, it also is computationally simple to implement.

The mean average absolute prediction errors are effectively the same as values obtained using much more accurate bedside testing kits during clinical trials [7]. This result illustrates the potential of this method for control applications. It also shows that the method does not introduce lag into the process as large time lags would result in much larger errors given the potential rapid variability of patient glucose dynamics in critical care [e.g. 7,15]. Note that the interstitial fluid to capillary blood lag in glucose level for CGMS has been reported to be 4-5 minutes [29], which should not prove significant given the errors involved and the limited amount by which glucose might change over this timeframe.

As a result, there is a significant potential clinical compromise between the advantages of integral-based methods in terms of computational cost, simplicity and convexity, and the clinical impact of the long 12-hour run-in period. Clinically, the impact is that for at least the first 8-10 hours of each sensor used poor results may be obtained. Given that device reported lifetimes are $\sim 3$ days approximately 1-2 sensors would be used per patient given average length of stays in many ICU studies of 3-5 days. Thus, the run-in period thus represents, on average, about $15 \%$ of the sensor lifetime, a significant portion of time to be less useful. Finally, it is also currently not clear that sensor lifetimes will grow longer as time and technology move forward. Hence, it is currently a conservative expectation that there would be 1-2 such run-in periods to contend with for the average patient and ICU. 
There are two possible clinical alternatives. First, an alternative method of control using bedside pin-stick based sensors could be used during this period. Such methods have recently been seen to be effective in critical care using hourly measurements [30,31]. A second approach would be to use other glycaemic control methods for the initial period, while overlapping the use of these sensors if possible during the run-in period on any following sensor use. Both approaches are obviously ad-hoc and other equally valid options may exist.

Therefore, the run-in period can significantly impact clinical usage. The main question thus remains one of whether the added data obtained enhances the clinical results, via whatever methods used in glycaemic control, versus using other methods and sensors. This clinical decision will likely vary greatly on the specific unit or patient circumstances. Finally, none of this discussion deals with the potentially more clinically important, and somewhat related, issue of detecting and managing sudden sensor failure or loss of calibration.

One limitation of this study is that measurements for these 17 subjects were primarily taken 2-3 hours apart, which can lead to dynamics being missed that could be captured with more frequent measurement. Such low measurement frequency may result in an "easier" problem for the methods presented. A second potential limitation of the overall analysis presented is that a model is used to both create and, via the methods presented, identify patient specific parameters, thus creating a situation where unmodelled dynamics do not exist outside the random error added. This problem is partly ameliorated by the prior clinical validation of the model and its reliance for the virtual patient profiles on measured clinical data. 
A third limitation is that the study does not consider errors due to poor, or absent, calibration of the CGMS sensor. Clinically, in such cases, the data would have much larger error and may not fully represent reality, which is difficult to model. In particular, there are currently no rigorously reported failure rates or modes of failure for these or similar emerging sensors. Thus, it is not yet possible, without direct clinical experience perhaps, to reliably and accurately model this behaviour. More specifically, failure prediction is the prediction of a random event, which this model does not include for two reasons. First, it is not known or reported what the random failure rate might be, if any. Second, the affect of random failure would likely be handled separately, by a safely implemented system, from the measurement noise filter method presented in this research.

For clinical control cases, such major failures or biased results would result in control algorithm failure, as the model would not be able to ascertain the patient's metabolic state. Hence, such a sensor failure is of clinical concern, but outside the scope of this study. Therefore, further clinical testing will have to be undertaken to delineate these issues and fully prove the algorithm presented by clinical studies. Thus, this work should primarily be considered as a proof of concept study presented as foundation for further, safe clinical study.

\section{CONCLUSIONS}

Tight glycaemic control in critical care, or any other situation, requires accurate measurements to achieve the best results. Emerging continuous glucose sensors, such 
as the Medtronic-Minimed CGMS device, offer significantly greater data density, and thus the potential for better control. However the measurements are subject to greater levels of inaccuracy than traditional pin-stick devices. This research presents a simple integral-based method for real-time control and filtering of glucose measurements with up to $40 \%$ added noise, using the CGMS device as a test case. While the analysis is based on critical care data, where glycaemic control is important, the methods presented can be readily generalised to applications outside critical care.

A noise model is presented that conservatively represents the error reported for CGMS devices. This model is used to create several virtual patient glucose profiles using retrospective patient data. The profiles are used to test the filtering methods presented using Monte Carlo simulations to statistically assess the performance measured in terms of one-hour (forward) prediction error. This approach mimics the role such a system would play in adaptive glycaemic control in critical care.

Overall results indicate that the methods presented achieve prediction errors of $6.2 \%$ +/- $5.2 \%$ on average with a range of $4.4-8.1 \%$ for the mean and $3.3-8.6 \%$ for the standard deviation. These results match or exceed the prediction performance obtained in a previous work for the same patient cohort using GlucoCard pin-stick sensors with $7-10 \%$ measurement error. Hence, the method presented is effective enough to show similar results to the current gold-standard for low-cost bedside glucose testing. Clinically, these results indicate that CGMS or similar emerging continuous glucose sensors have significant potential, in current form, to achieve tight, clinical glucose control. 


\section{REFERENCES}

[1] Capes, SE Hunt, D Malmberg, K Gerstein, HC. Stress Hyperglycaemia and Increased Risk of Death after Myocardial Infarction in Patients with and without Diabetes: A Systematic Overview, Lancet, 355 (9206) (2000) 773-778.

[2] Van den Berghe, G Wouters, P Weekers, F Verwaest, C Bruyninckx, F Schetz, M Vlasselaers, D Ferdinande, P Lauwers, P Bouillon, R. Intensive Insulin Therapy in the Critically Ill Patients, N Engl J Med. 345 (19) (2001) 1359-1367.

[3] Mizock, BA Alterations in Fuel Metabolism in Critical Illness: Hyperglycaemia, Best Pract Res Clin Endocrinol Metab. 15 (4) (2001) 533-551.

[4] McCowen, KC Malhotra, A Bistrian, BR Stress-Induced Hyperglycemia, Crit Care Clin, 17 (1) (2001) 107-124.

[5] Bistrian, BR. Hyperglycemia and Infection: Which Is the Chicken and Which Is the Egg? JPEN J Parenter Enteral Nutr. 25 (4): (2001) 180-181.

[6] Krinsley, JS. Association between Hyperglycemia and Increased Hospital Mortality in a Heterogeneous Population of Critically Ill Patients, Mayo Clin Proc. 78 (12) (2003) 1471-1478.

[7] Chase, JG, Shaw, GM, Lin, J, Doran, CV, Hann, CE, Robertson, MB, Browne, PM, Lotz, T, Wake, GC and Broughton, R (2005). "Adaptive bolus-based targeted glucose regulation of hyperglycaemia in critical care," Medical Engineering and Physics, Vol 27(1), pp. 1-11.

[8] A.M. Albisser, B.S. Leibel, T.G. Ewart, Z. Davidovac, C.K. Botz, W. Zingg, H. Schipper, R. Gander, Clinical Control of Diabetes by the Artificial Pancreas, Diabetes. 23 (5) (1974) 397-404.

[9] Hann, CE, Chase, JG, Lin, J, Lotz, T, Doran, CV, and Shaw, GM (2005). "Integral-Based Parameter Identification For Long-Term Dynamic Verification Of A Glucose-Insulin System Model,” J. of Computer Methods and Programs in Biomedicine, Vol 77(3), pp. 259-270.

[10] The Diabetes Research in Children Study Group (DIRECNET) (2004). "Accuracy of the GlucoWatch G2 Biographer and the Continuous Glucose Monitoring System During Hypoglycemia," Diabetes Care, Vol 27, pp. 722-726.

[11]Goldberg, P, Siegel, M, Russell, R, Sherwin, R, Halickman, J, Cooper, D, Dziura, J and Nzucchi, S (2004). "Experience with the Continuous Glucose Monitoring System in a Medical Intensive Care Unit," Diabetes Technology and Therapeutics, Vol. 6(3), pp. 339-347.

[12] Tavris, DR, Shoaibi, A (2004). "The public health impact of the MiniMed Continuous Glucose Monitoring System (CGMS) - an assessment of the literature," Diabetes Technology and Therapeutics, Vol 6(4),pp. 518-522.

[13]Chee, F, Fernando, T and van Heerden, PV (2003). "Closed Loop Glucose Control in Critically Ill Patients Using Continuous Glucose Monitoring System (CGMS) in Real Time," IEEE Trans on Information Technology in Biomedicine, Vol. 7(1), pp. 43-53.

[14] Vreisendorp, TM, Devries, JH, Holleman, F, Dzoljic, M and Hoekstra, JBL (2005). "The use of two continuous glucose sensors during and after surgery," Diabetes Technology and Therapeutics, Vol. 7(2), pp. 315-322.

[15] Raskin, P (2005). "Disadvantages of continuous glucose monitoring," Diabetes Technology and Therapeutics, Vol. 7(2), pp. 358-359.

[16] Nicholls, JH (2005). "The evolution of glucose meters," Diabetes Technology and Therapeutics, Vol. 7(2), pp. 295-297. 
[17] Wolpert, HA (2005). "Use of continuous glucose monitoring technology," Diabetes Technology and Therapeutics, Vol. 7(2), pp. 360.

[18] Steil, GM (2005). "Closed loop blood glucose control using a physiologic insulin delivery algorithm - clinical results," Diabetes Technology and Therapeutics, Vol. 7(2), pp. 359.

[19] Steil, GM, Panteleon, AE and Rebrin, K (2004). "Closed-loop insulin deliverythe path to physiological glucose control," Advanced Drug Delivery Reviews, Vol. 56(2), pp.125-44.

[20]Chase, JG, Shaw, GM, Lin, J, Doran, CV, Hann, CE, Robertson, MB, Brown, PM, Lotz, T, Wake, GC and Broughton, B (2004). “Adaptive Bolus-Based SetPoint Regulation of Hyperglycemia in Critical Care," Proc $26^{\text {th }}$ International Conf of IEEE Engineering in Med and Biology Society (EMBS 2004), San Francisco, CA, Sept 1-5, pp. 3463-3466.

[21]Chase, JG, Shaw, GM, Lin, J, Doran, CV, Bloomfield, M, Wake, GC, Broughton, B, Hann, C and Lotz, T (2005). "Impact of insulin-stimulated glucose removal saturation on dynamic modelling and control of hyperglycaemia," Intl Journal of Intelligent Systems Technologies and Applications (IJISTA), Vol 1(1-2), pp. 7994.

[22] Wong, XW, Shaw, GM, Chase, JG, Hann, CE, Lotz, T and Lin, J (2005). “Active insulin control with variable nutrition for targeted glucose control in critically ill patients,” New Zealand Medical Journal (NZMJ), Technical Note, Vol 118(1215), online journal, www.nzma.org.nz.

[23] P. Vicini, A. Caumo, C. Cobelli, The Hot IVGTT Two-Compartment Minimal Model: Indexes of Glucose Effectiveness and Insulin Sensitivity, Am J Physiol. 273 (5 Pt 1) (1997) E1024-1032.

[24]R.A. DeFronzo, J.D. Tobin, R. Andres, Glucose Clamp Technique: A Method for Quantifying Insulin Secretion and Resistance, Am J Physiol. 237 (3) (1979) E214-223.

[25] Clarke, WL, Becker, DJ, Cox, D, Santiago, JV, White, NH, Betschart, J, Eckenrode, K, Levandoski, LA, Prusinski, EA and Simineiro, LM (1988). "Evaluation of a new system for self blood glucose monitoring," Diabetes Res Clin Practice, Vol. 4(3), pp. 209-213.

[26] C.V. Doran (2004). Modelling and Control of Hyperglycaemia in Critical Care Patients, Masters of Engineering Thesis, Mechanical Engineering, University of Canterbury, Christchurch, New Zealand.

[27] Buckingham, B (2005). "Advantages of near-continuous glucose monitoring," Diabetes Technology and Therapeutics, Vol. 7(2), pp. 347.

[28] Knobbe, EJ and Buckingham, B (2005). "The extended Kalman filter for continuous glucose monitoring," Diabetes Technology and Therapeutics, Vol. 7(1), pp. 15-27.

[29] Steil, GM, Rebrin, K, Mastrototaro, J, Bernada, B and Saad, MF (2003). "Detemination of plasma glucose during rapid glucose excursions with a subcutaneous glucose sensor," Diabetes Technology and Therapeutics, Vol. 5(1), pp. 27-31.

[30] Chase, JG, Lonergan, T, LeCompte, A, Willacy, M, Shaw, GM, Wong, XW, Lin, J, Lotz, TS and Hann, CE (2005). "Tight glucose control in critically ill patients using a specialized insulin-nutrition-table,” $12^{\text {th }}$ Intl Conf on Biomedical Eng. (ICBME), Singapore, Dec 7-10. 
[31] Chase, JG, Wong, XW, Shaw, GM, Hann, CE Lin, J, and Lotz, TS (2005).

"Clinical trials of active insulin and nutrition control in critically ill patients," $12^{\text {th }}$ Intl Conference on Biomedical Engineering (ICBME), Singapore, Dec 7-10. 
TABLE I: Patient cohort and data

\begin{tabular}{|c|c|c|c|c|c|c|}
\hline $\begin{array}{l}\text { Patient } \\
\text { Number }\end{array}$ & $\begin{array}{l}\text { Medical } \\
\text { Subgroup }\end{array}$ & $\begin{array}{l}\text { APACHE } \\
\text { II Score }\end{array}$ & Age & Sex & Mortality & Diabetes \\
\hline 24 & $\begin{array}{c}\text { Other } \\
\text { Medical }\end{array}$ & 25 & 47 & M & $\mathrm{Y}$ & Type 1 \\
\hline 87 & $\begin{array}{c}\text { Other } \\
\text { Medical }\end{array}$ & 26 & 62 & $\mathrm{~F}$ & & \\
\hline 130 & Trauma & 11 & 21 & $\mathrm{M}$ & & Type 1 \\
\hline 229 & Cardiac & 15 & 73 & $\mathrm{~F}$ & & \\
\hline 289 & Cardiac & 18 & 70 & $\mathrm{M}$ & & \\
\hline 468 & $\begin{array}{l}\text { General } \\
\text { Surgical }\end{array}$ & 32 & 76 & M & & \\
\hline 484 & $\begin{array}{c}\text { Other } \\
\text { Medical }\end{array}$ & 34 & 30 & $\mathrm{~F}$ & & \\
\hline 486 & $\begin{array}{l}\text { General } \\
\text { Surgical }\end{array}$ & 22 & 76 & $\mathrm{~F}$ & & Type 2 \\
\hline 519 & $\begin{array}{l}\text { General } \\
\text { Surgical }\end{array}$ & 29 & 69 & M & & Type 2 \\
\hline 554 & $\begin{array}{c}\text { Other } \\
\text { Medical }\end{array}$ & 26 & 20 & $\mathrm{~F}$ & & Type 1 \\
\hline 666 & Cardiac & 8 & 44 & $\mathrm{~F}$ & & Type 2 \\
\hline 847 & $\begin{array}{c}\text { Other } \\
\text { Medical }\end{array}$ & 17 & 67 & $\mathrm{~F}$ & & \\
\hline 1016 & $\begin{array}{l}\text { General } \\
\text { Surgical }\end{array}$ & 20 & 37 & $\mathrm{~F}$ & & Type 2 \\
\hline 1025 & Pulmonary & 36 & 48 & $\mathrm{M}$ & & Type 2 \\
\hline 1090 & $\begin{array}{l}\text { General } \\
\text { Surgical }\end{array}$ & Unknown & 37 & F & & \\
\hline 1099 & Pulmonary & Unknown & 24 & $\mathrm{M}$ & $\mathrm{Y}$ & \\
\hline 1125 & $\begin{array}{c}\text { Other } \\
\text { Medical }\end{array}$ & Unknown & 72 & $\mathrm{~F}$ & $\mathrm{Y}$ & \\
\hline
\end{tabular}


Table II: Correlation coefficients for 7 patients over $\mathrm{N}=20$ simulations

\begin{tabular}{|c|r|}
\hline $\begin{array}{l}\text { Patient } \\
\text { Number }\end{array}$ & $\begin{array}{l}\text { Correlation } \\
\text { Coefficient }\end{array}$ \\
\hline 24 & 0.68 \\
87 & 0.86 \\
130 & 0.85 \\
486 & 0.82 \\
519 & 0.75 \\
554 & 0.88 \\
1025 & 0.81 \\
\hline Average & $\mathbf{0 . 8 1}$ \\
\hline
\end{tabular}


Table III: 1-hour forward glucose prediction error results where GlucoCard results are from Hann et al [9] for comparison.

\begin{tabular}{|c|c|c|c|c|}
\hline Patient & \multicolumn{2}{|c|}{$\begin{array}{c}\text { Mean Prediction Error (\%) } \\
\text { Smoothed }\end{array}$} & \multicolumn{2}{|c|}{$\begin{array}{c}\text { Error Standard Deviation } \\
(\%)\end{array}$} \\
\hline 24 & 5.86 & 4.97 & 4.00 & 3.89 \\
\hline 87 & 4.71 & 5.03 & 5.21 & 4.08 \\
\hline 130 & 10.12 & 7.66 & 9.55 & 6.17 \\
\hline 229 & & 5.85 & & 5.17 \\
\hline 289 & & 7.04 & & 5.84 \\
\hline 468 & & 6.31 & & 4.96 \\
\hline 484 & & 4.66 & & 3.58 \\
\hline 486 & & 7.88 & & 7.44 \\
\hline 519 & 5.25 & 5.52 & 5.98 & 4.86 \\
\hline 554 & 10.90 & 8.06 & 8.89 & 8.55 \\
\hline 666 & 4.66 & 5.46 & 3.01 & 4.53 \\
\hline 847 & & 7.43 & & 5.44 \\
\hline 1016 & 7.01 & 6.14 & 6.27 & 5.36 \\
\hline 1025 & 5.09 & 6.27 & 4.54 & 6.25 \\
\hline 1090 & 1.86 & 4.37 & 0.87 & 3.26 \\
\hline 1099 & & 6.97 & & 5.13 \\
\hline 1125 & 6.83 & 5.71 & 4.78 & 4.19 \\
\hline Average & 6.23 & 6.20 & 5.31 & 5.22 \\
\hline
\end{tabular}



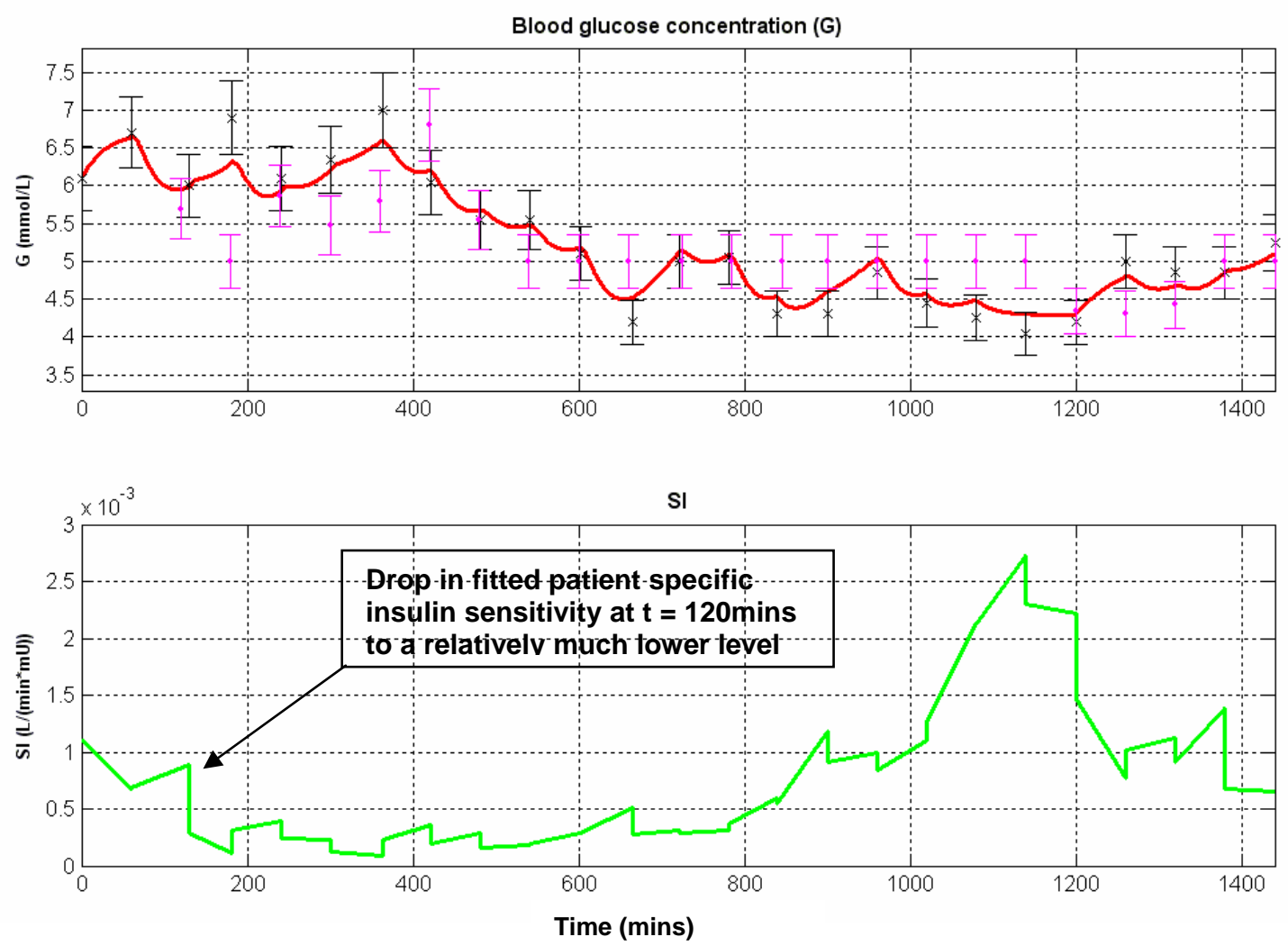

Figure 1: Example of a 24-hour clinical trial result showing modelled and measured

(x) glucose values and fitted insulin sensitivity, SI. Circled (o) data points show hourly glycaemic targets. The error bars for all cases show the 7\% measurement error. The trial uses the adaptive control methods from Chase et al [7]. 


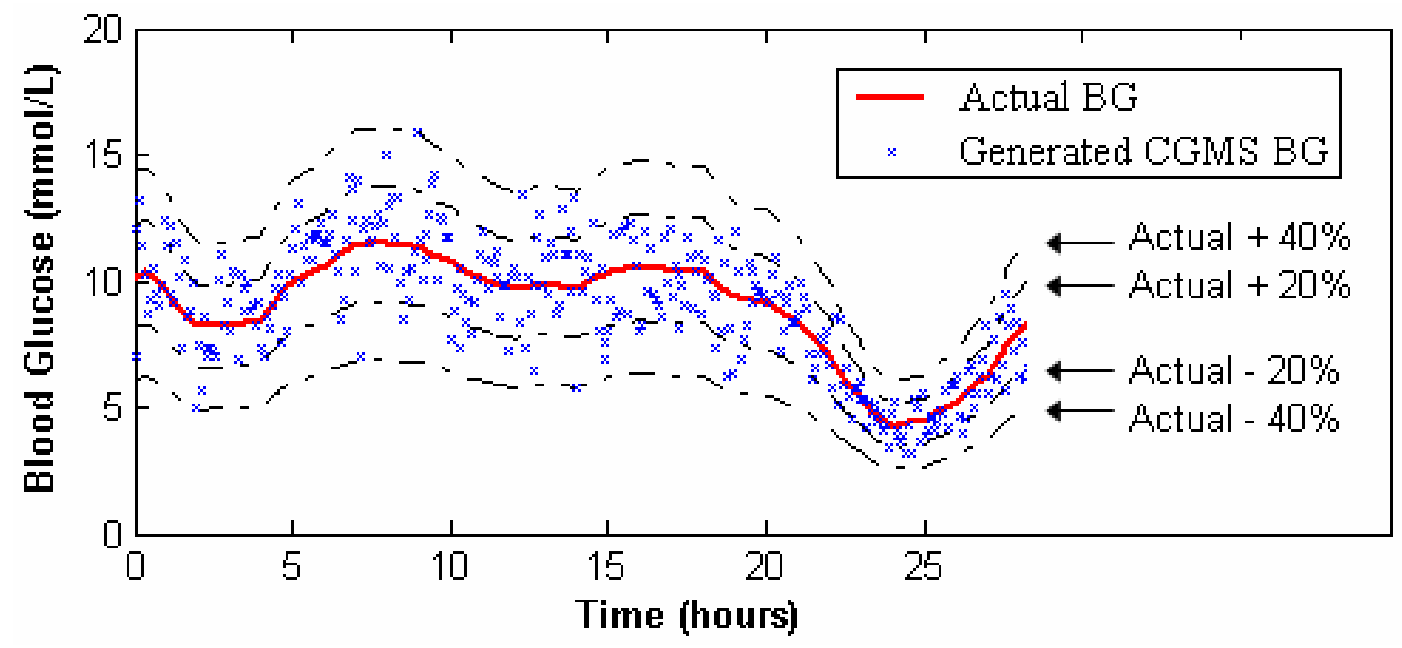

Figure 2: Example of approximated CGMS error to a simulated glucose profile.

Dashed lines show $20 \%$ and $40 \%$ bounds to estimate the magnitude of any error. 


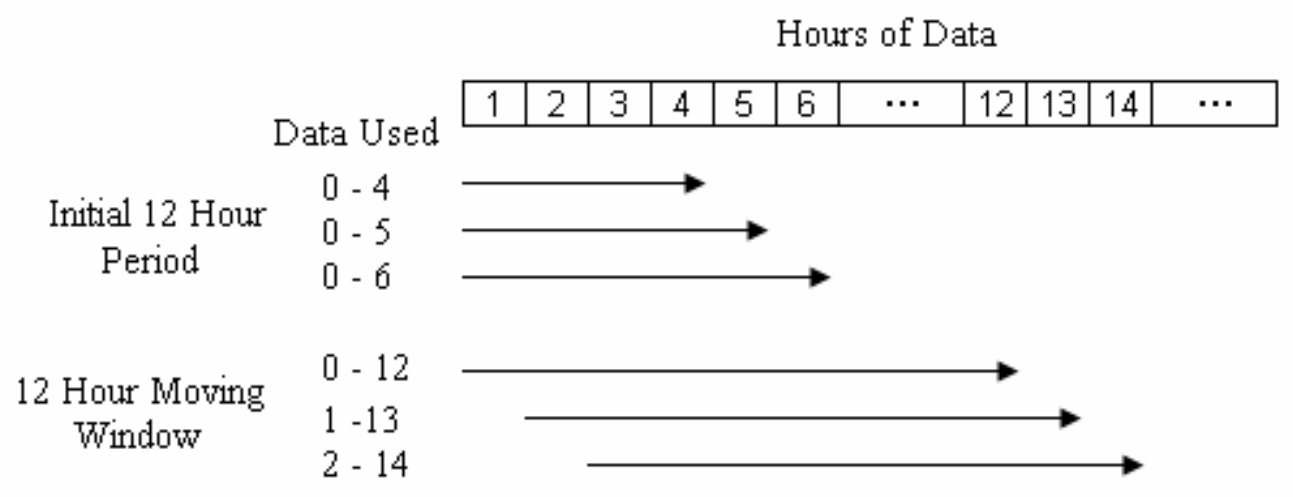

Figure 3: Identification and smoothing algorithm data window. 


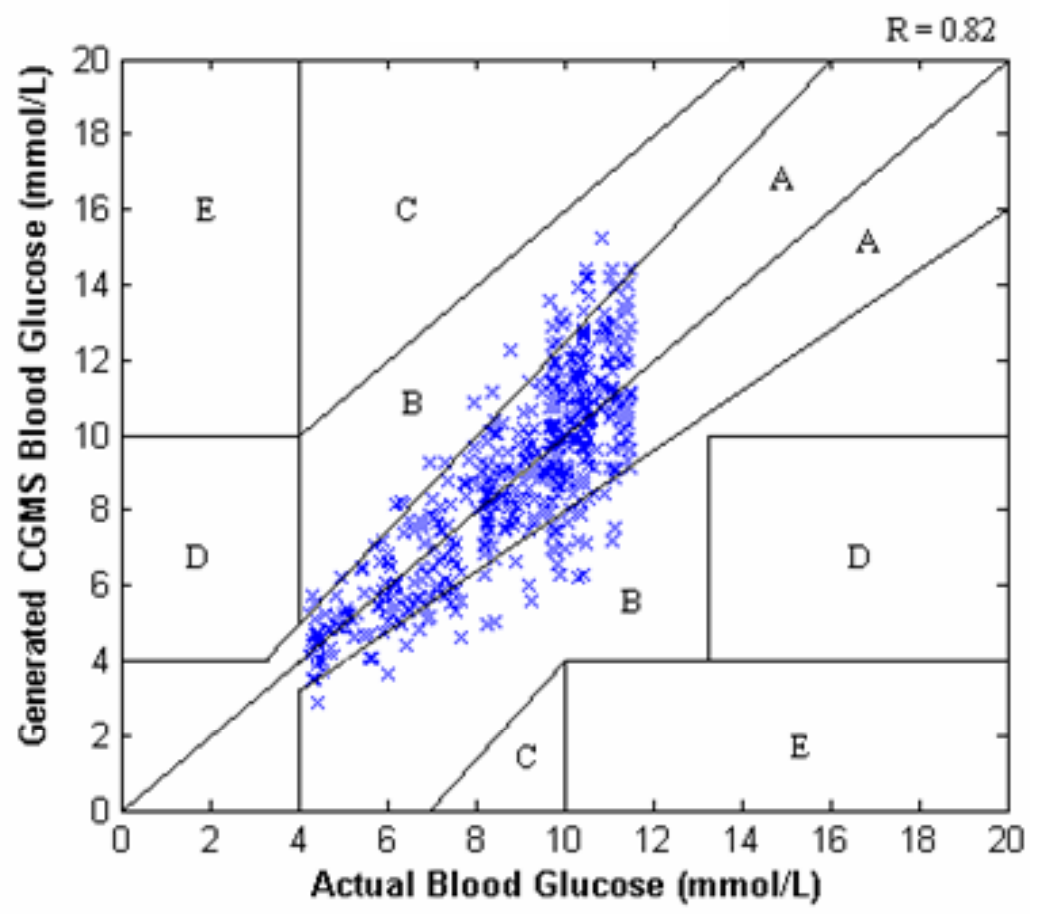

Figure 4: Clarke Error Grid for 1 of the 17 patient simulations, with $\mathrm{R}=0.82$ over all $\mathrm{N}=20$ Monte Carlo simulations for that patient 


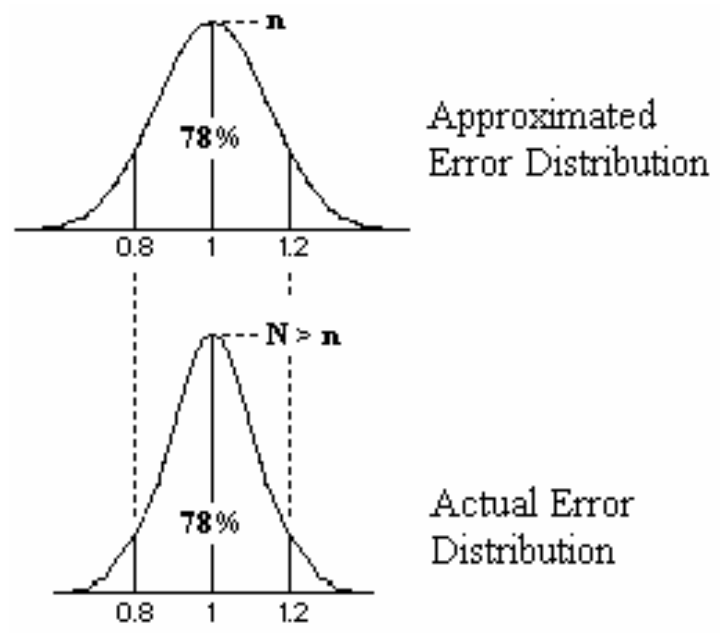

Figure 5: Possible actual distribution versus normal approximated error distribution 